\title{
Literarische Männlichkeitsentwürfe in Christian Krachts Imperium und Steffen Kopetzkys Risiko
}

\section{Literary Concepts of Masculinity in Christian Kracht's Imperium and Steffen Kopetzyk's Risiko}

\author{
Saniye UYSAL ÜNALAN ${ }^{1}$ [
}

${ }^{1}$ Assoc. Dr., Ege University, Faculty of Letters, German Language and Literature Department, Izmir, Turkey

\section{ORCID: S.U.Ü. 0000-0002-4117-7158}

\section{Corresponding author:} Saniye UYSAL ÜNALAN, Ege Üniversitesi, Edebiyat Fakültesi Alman Dili ve Edebiyatı Bölümü, İzmir, Türkiye E-mail: suysalunalan@gmail.com

Submitted: 02.03 .2020

Revision Requested: 27.03.2020

Last Revision Received: 08.04.2020

Accepted: 09.04.2020

Citation: Uysal Unalan, S. (2020). Literarische Männlichkeitsentwürfe in Christian Krachts Imperium und Steffen Kopetzkys Risiko. Alman Dili ve Edebiyatı Dergisi - Studien zur deutschen Sprache und Literatur, 43, 155-185.

https://doi.org/10.26650/sds|2020-0006

\begin{abstract}
DEUTSCH)
Die Romane Imperium von Christian Kracht und Risiko von Steffen Kopetzky können als historische Abenteuerromane bezeichnet werden, die sich mit der Zeit des Wilhelminischen Deutschland auseinandersetzen. Die zeitliche bzw. kulturgeschichtliche Verortung der erzählten Welt beider Romane im Kontext des Kolonialismus ist hierbei von besonderer Wichtigkeit. Anhand der spezifischen Konfiguration der männlichen Träger bzw. Akteure des Kolonialismus eröffnen diese Texte eine dezidiert kritische Perspektive, die sich vor allem auf eine grundsätzliche Infragestellung der mit Solidität, Selbstbehauptung, Kontrolle sowie Macht enggeführten Männlichkeitskonzipierung des Wilhelminischen Deutschland bezieht. In der vorliegenden Untersuchung wird das Argument stark gemacht, dass Krachts Imperium wie auch Kopetzkys Risiko insbesondere im Hinblick auf die Konstruktion der männlichen Heldenfiguren sowie der damit verknüpften Handlungs- und Erfahrungswelten als solche Narrative beschreibbar werden, in denen tradierte Bilder und Diskurse von heldenhafter Männlichkeit vehement in Frage gestellt werden. Vor dem Hintergrund der literaturwissenschaftlich begründeten Männlichkeitsforschung werden die fiktionalen Bilder und Konfigurationen von Männlichkeit in den Fokus genommen. Der kurzen Nachzeichnung der kulturellen Diskurse über Männlichkeit zu Beginn des 20. Jahrhunderts folgt die Analyse der Männerfiguren. Dabei wird die diskursive Verknüpfung der fiktionalen Konfiguration von Männlichkeit mit der imperialen Weltpolitik Deutschlands veranschaulicht. Anschließend konzentriert sich die Arbeit auf die Signifikanz des Körpers, anhand dessen die ambivalente Verfasstheit von kolonialer Männlichkeit und die Kritik an der Kolonialpolitik Deutschlands pointiert zum Ausdruck kommen. Festgestellt werden konnte in diesem Zusammenhang, dass die in beiden Romanen konfigurierten Männlichkeitsbilder unmittelbar im Zeichen des ins Negative umschlagenden Kolonialismus-Diskurses stehen und gerade daher als dessen Negativfolie fungieren
\end{abstract}

Schlüsselwörter: Imperium, Christian Kracht, Risiko, Steffen Kopetzky, literarische Männlichkeitsentwürfe 


\begin{abstract}
ENGLISH)
Christian Kracht's Imperium and Steffen Kopetzky's Risiko can be described as historical adventure novels, which deal with the Wilhelminian Era. In both novels it is significant that the context of the German colonialism plays an important role. Closely related to the specific configuration of the male subjects of colonialism, these novels open up a critical perspective on the typical perception of masculinity of the Wilhelminian Era, in which masculinity is associated with solidity, selfassertion, control and force. The aim of this study is to demonstrate that Kracht's Imperium as well as Kopetzky's Risiko can be seen as narratives which vehemently question conventional images and discourses of heroic masculinity. The study refers to literary studies which focus on the literary conception of masculinity. After a short outline of the cultural discourses about masculinity at the beginning of the $20^{\text {th }}$ century, I will analyze the male figures. The illustration of the discursive link between the fictional configuration of masculinity and the imperial world politics of Germany constitutes a crucial point of the analysis. Therefore, I will also concentrate on the significance of the body. In this sense, it could be ascertained that the images and configuration of masculinity in Kracht's Imperium and in Kopetzky's Risiko are dominated by colonial discourses, which turn out to be negative. So, the literary conception of masculinity serves as a critical dimension, that reveals the negative aspects of German colonialism politics in the Wilhelminian Era.
\end{abstract}

Keywords: Imperium, Christian Kracht, Risiko, Steffen Kopetzky, literary concepts of masculinity

\title{
EXTENDED ABSTRACT
}

Christian Kracht's Imperium and Steffen Kopetzky's Risiko can be described as historical adventure novels, which deal with the Wilhelminian Era. While Kracht's Imperium presents the eccentric life story and visions of the historical figure August Engelhardt who travels to the South Pacific, Kopetzky's Risiko deals with the historical German-Ottoman expedition to Afghanistan during World War I. In both novels it is significant that the context of German colonialism plays an important role. The travel of the male figures to an exotic topography, the associated adventures and strains as well as the embedding of these aspects in the context of colonialism take on a special meaning for the argumentation of this study. Closely related to the specific configuration of the male subjects such as actors of colonialism, these novels open up a critical perspective on the typical perception of masculinity of the Wilhelminian Era, in which masculinity is explicitly associated with solidity, self-assertion, control and force. With regard to the construction of the male figures and their experiences, the aim of this study is to demonstrate that Kracht's Imperium and Kopetzky's Risiko can be seen as narratives which vehemently question and deconstruct conventional images and discourses of heroic masculinity. Based on the assumption that these novels contain postcolonial aspects and references to the political discourses and the colonial context of the Wilhelminian Era, this study will focus on the fictional images and configurations of the male figures in both novels. In this context, the study refers not only to literary studies which focus on the literary conception and configuration of masculinity, but also to sociological categories such as hegemonic and marginalised masculinity. In doing so, the fictional images and configurations of 
masculinity that can be observed in both novels will be related to the discourses of the Wilhelminian Era. After a short outline of the cultural discourses about masculinity at the beginning of the $20^{\text {th }}$ century the study concentrates on an analysis of the male figures of the novels. The ideals of maleness and power particularly represented by Wilhelm II have been read as a form of male self-assertion and thus of hegemonic masculinity, which also correlates with the military and cultural expansion policy of that time. This very point constitutes the referential framework for the configuration and conceptualization of the male figures such as August Engelhardt in the novel Imperium or Oskar Niedermayer and Sebastian Stichnote in the novel Risiko. So, the illustration of the discursive link between the fictional configuration of masculinity and the imperial world politics of Germany constitutes a crucial point of the analysis. Therefore, the study concentrates on the significance of the body. Moreover, it becomes clear that both novels criticize the colonial ambitions of Germany by reducing the male characters to their bodies and simultaneously reveal the ambivalent character of colonial masculinity. In this sense, it could be ascertained that the images and configuration of masculinity in Kracht's Imperium as well as in Kopetzky's Risiko are dominated by the colonial discourses which turn out to be negative. So, the literary conception of masculinity serves as a critical dimension that reveals the negative aspects of German colonialism politics in the Wilhelminian Era. The utopia of August Engelhardt turns into in a barbaric cannibalism, which can be regarded as an anticipation of Nazi-Germany. Meanwhile the actors of the expedition reach the very limits of a human being, for they experience a significant reduction in bodies only. From this perspective the study makes clear that both novels undermine not only the myth of colonialism, but also the concept of the white man through the presented stories of important historical and fictional male figures. 


\section{Einleitung}

Der gemeinsame Nenner der beiden Romane Imperium von Christian Kracht und Risiko von Steffen Kopetzky dürfte darin zu sehen sein, dass es sich bei beiden Texten um historische Abenteuerromane handelt, die sich mit der Zeit des Wilhelminischen Deutschland auseinandersetzen. Folgerichtig finden die imperialen bzw. kolonialen Interessen des Deutschen Kaiserreichs sowie die damit unmittelbar verknüpften historischen Hintergründe Eingang in diese Romane. Während Imperium ${ }^{1}$ die exzentrische Lebensgeschichte von August Engelhardt, einem deutschen Aussteiger und Anhänger der Lebensreform-Bewegung um 1900, und dessen auf die Kokosnuss gegründete neue Lebensweise $^{2}$ in ironischer Manier und mit zahlreichen intertextuellen Verweisen ${ }^{3}$ erzählt, befasst sich Risiko mit einer deutschen Expedition nach Afghanistan unter der Leitung von Oskar Niedermayer zu Beginn des Ersten Weltkriegs. Dabei kommt der kulturgeschichtlichen Verortung der erzählten Welt beider Romane im Kontext des deutschen Kolonialismus eine besondere Bedeutung zu. Die Romane Imperium und Risiko eröffnen insofern eine dezidiert kritische Perspektive auf die Machtdiskurse des Deutschen Kaiserreichs, als die von Kaiser Wilhelm II. repräsentierte Imperialpolitik und Männlichkeitsinszenierung, bei der Männlichkeit weitgehend mit Solidität, Selbstbehauptung, Kontrolle sowie Macht enggeführt wird, einer grundsätzlichen Infragestellung unterzogen wird. Denn wenngleich Imperium und Risiko als Abenteuerromane $^{4}$ lesbar sind, ist es doch bezeichnend, dass sie insbesondere im Hinblick auf die Konstruktion ihrer männlichen Heldenfiguren sowie der damit verknüpften Handlungs- und Erfahrungswelten als solche Narrative beschreibbar werden, in denen tradierte Bilder und Diskurse von hegemonialer Männlichkeit und zusammenhängend damit die Machtdiskurse des Wilhelminischen Deutschland vehement in Frage gestellt werden. Ausgehend von der Einsicht, dass diese beiden Texte

1 Es sei darauf hingewiesen, dass dieser Roman in der deutschen Literaturkritik für große Debatten sorgte, in denen es vorwiegend um das vermeintlich rassistische Denken des Autors ging und somit eigentlich um die Aufhebung der innerhalb der literaturwissenschaftlichen Praxis als selbstverständlich gesehenen Trennung von Fiktion und Autor. Siehe dazu den Band von Winkels (2015). So bemerkt Schumacher, dass der Roman durch ein irritierendes Verfahren in seiner Erzählweise gekennzeichnet sei. Das „Verwirrspiel zwischen Fakt und Fiktion" sei ein "Strukturprinzip, das nicht nur Orte, Zeiten und Figuren betrifft und sich nicht nur auf Verschiebungen und Überlagerungen der Grenzen zwischen Fakt und Fiktion beschränkt, sondern ganz grundlegend Krachts Schreibverfahren und Erzählweise prägt" (Winkels, 2015, S. 136).

2 Zu dieser historischen Figur vgl. Wendt (2013, S. 47-48) und Hauenstein (2014, S. 123-126).

3 Zu den im Roman enthaltenen intertextuellen Verweisen siehe Birgfeld (2012), Hauenstein (2014, S. 129134), Schumacher (2015, S. 139-142) oder Moll (2017, S. 146 und 157).

4 Siehe dazu beispielsweise die Verlagswerbung beider Romane: Klett Cotta (2020) und Kiepenheuer \& Witsch (2020). 
eine postkoloniale Perspektive auf die deutsche Geschichte des Kolonialismus und Imperialismus eröffnen ${ }^{5}$, geht die vorliegende Untersuchung somit der Frage nach, welches Verhältnis zwischen den literarischen Männlichkeitsentwürfen und den Strukturen bzw. Ideologien des deutschen Kolonialismus festzustellen ist. Auf diese Weise wird es an diesen Beispielen möglich, mit Blick auf die literarische Konfiguration von Männlichkeit den Gender-Aspekt von literarischen Texten herauszuarbeiten, denen ein postkoloniales Potential immanent ist. Ein solches Potential lässt sich offensichtlich als eine Kritik am abendländischen Denken bzw. an eurozentrischen Paradigmen beschreiben, die bekanntlich männlich codiert sind und somit auch mit männlicher Hegemonie einhergehen ${ }^{6}$. Gerade an diesem Punkt knüpft die vorliegende Untersuchung an, denn es ist die spezifische Konfiguration der männlichen Träger bzw. Akteure des Kolonialismus, anhand derer die Affinität von Männlichkeit und Kolonialismus nachweisbar wird. Dabei stellt sich vor allem die Frage, welche Formen von Männlichkeit postkoloniale Literatur zu konzipieren vermag, wenn es um die Kritik oder gar Dekonstruktion von männlicher Macht geht. Um diese Fragen beantworten zu können, sollen im Folgenden zunächst grundlegende Aspekte zur literaturwissenschaftlich begründeten Männlichkeitsforschung vorgestellt werden. Dabei wird es darum gehen, die diskursive Gemachtheit von Männlichkeit zu erläutern und einen kurzen Einblick in die Diskurse von Männlichkeit zu Beginn des 20. Jahrhunderts zu verschaffen. Insbesondere unter Bezugnahme auf die von R. W. Connell (2012) etablierten soziologischen Beschreibungskategorien der "hegemonialen“ und „marginalisierten" Männlichkeit, die der Literaturwissenschaftler Toni Tholen für die Analyse von Männlichkeit in der Literatur fruchtbar gemacht hat, soll anschließend die Konfiguration der Männerfiguren beider Romane näher in den Blick genommen werden. Gezeigt werden soll in diesem Zusammenhang, wie der Übergang von hegemonialer zur marginalisierter Männlichkeit in diesen Romanen inszeniert wird und welches kritische Potential damit einhergeht.

5 Zur postkolonialen Lektüre von Krachts Imperium vgl. Hauenstein (2014, S. 120-151); Dürbeck (2014) sowie Uysal Ünalan (in Vorbereitung). Zur Analyse des Romans Risiko aus postkolonialer Sicht vgl. Uysal Ünalan (2019).

6 So besteht auch insofern eine Affinität zwischen postkolonialen und Gender-Ansätzen, als beide von einer "strukturelle[n] Machtdifferenz" ausgehen: „Postkoloniale Theorie greift den Ethno- und Eurozentrismus des abendländischen Denkens an", wohingegen Gender-Theorien "patriarchale Macht und andere Formen ,männlicher Herrschaft" (Dietze, 2005, S. 307) angreifen. Auch die literaturwissenschaftliche Erforschung von Männlichkeit verpflichtet sich diesem Anliegen, insofern als sie nicht-hegemoniale Formen von literarischer Männlichkeit in den Blick nimmt (vgl. Tholen, 2007, S. 472). 


\section{Männlichkeit als Konstruktion}

Innerhalb der symbolischen Geschlechterordnung wird Männlichkeit mit "Geistigkeit und Kultur" zusammengedacht, währenddessen werden Körper und Natur „als ,weiblich” codiert" (Braun \& Stephan, 2005, S. 7). Dabei handelt es sich Braun und Stephan zufolge zweifelsfrei um eine "Zuordnung, die sich bis weit in die Moderne hinein fortgesetzt hat und noch heute prägend bleibt für die Art, wie über ,weibliche Irrationalität', Unberechenbarkeit und davon abgeleitet ,Unwissenschaftlichkeit' gesprochen wird" (Braun \& Stephan, 2005, S. 7). Somit bildet im Rahmen der symbolischen Geschlechterordnung die Zusammenführung von Männlichkeit mit Rationalität bzw. Wissenschaftlichkeit die Grundlage für die Überlegenheit des männlichen Geschlechts wie auch dessen hierarchischem Verhältnis zum weiblichen Geschlecht. So zeigt auch ein Blick auf das Wortfeld des Begriffs,männlich', dass dessen Herkunft auf die Bedeutungen „dem Mann angemessen; tapfer, mutig“ zurückgeht (Duden Etymologie, 1997, S. 439)7. In ihrer Untersuchung zur Geschichte dieses Begriffs legt Ute Frevert (vgl. 1995, S. 32) dar, dass insbesondere im 19. Jahrhundert in Wörterbüchern die Tendenz zu beobachten sei, den Begriff,Mann' vornehmlich im Verhältnis und in Abgrenzung zur,Frau'zu definieren. Dabei werde vor allem der "männliche[] Charakter" akzentuiert, der von der anatomischen wie auch physiologischen Bestimmung der männlichen Existenz abgeleitet werde. Dabei sei "das weibliche Geschlecht" stets „Bezugs- und Vergleichseinheit [...], von dem man sich abzusetzen und das man zu übertreffen" (Frevert, 1995, S. 32) suche. Seinen Niederschlag finde dieser Umstand in solchen Definitionen des Begriffs,Mann', in denen der "Gebrauch des Komparativs" besonders hervortrete, wie etwa "höherer Mut, stärkeres Kraftgefühl, höheres Vermögen an Kraft, das vermögendere Geschlecht" (Frevert, 1995, S. 32, Hervorhebungen im Original). Vor diesem Hintergrund konstatiert Frevert, dass "die von bürgerlichen Autoren für bürgerliche Leser geschriebenen Konversations- und Sachlexika“ gewissermaßen bis „ins 20. Jahrhundert hinein“ folgende Devise proklamieren: „Dem Manne der Staat, der Frau die Familie!“ (Frevert, 1995, S. 59). Diskursive Bestimmungen dieser Art denken den Mann also mit Öffentlichkeit, Macht und Politik zusammen, wohingegen diese im Gegenzug auf die Exklusion und Einschließung der Frau in den privaten Bereich bedacht sind. Dabei ist der dekretierende sowie normative Gestus dieser symbolischen Geschlechterordnung bemerkenswert, die vor allem im Rahmen der Geschlechterforschung ins kritische Visier gerät. In diesem Zusammenhang ist es vor allem der um die Jahrhundertwende von Sigmund Freud

7 Siehe dazu auch Vahsen (2002, S. 252). 
begründete Diskurs über Weiblichkeit, der einer kritischen Relektüre unterzogen wurde. Nach Inge Stephan handelt es sich in der Psychoanalyse um "Verlagerungen" der männlichen Psyche und Befindlichkeiten auf das weibliche Geschlecht, so dass davon auszugehen sei, dass nicht die Frau das "Rätsel“ sei, sondern der Mann, der "in den toten Winkel der Aufmerksamkeit geriet" (Stephan, 2003, S. 12). Denn Freuds Projekt der Psychoanalyse, das sich bekanntlich die Aufklärung des menschlichen Unterbewusstseins zur Aufgabe gemacht hat, codiert das Konzept,Weiblichkeit' als „Rätsel“ (Freud, 1999a, S. 120). Gerade dies verweist auf den Umstand, dass diese Codierung mit der autoritativen Instanz in Bezug gesetzt werden muss, die diese Aussage überhaupt zur Artikulation bringt. Das bedeutet also im Umkehrschluss, dass das mit Weiblichkeit korrelierte Rätselhafte vielmehr auf den Mann bezogen ist. Diesbezüglich formuliert Inge Stephan Folgendes: „Die Frage ,Was will das Weib?' lenkte von der eigentlichen Frage ab: Was will der Mann bzw. was ist der Mann?" (Stephan, 2003, S. 12) ${ }^{8}$. Im Kontext der kulturwissenschaftlich fundierten Geschlechterforschung besteht mittlerweile weitgehend Konsens darüber, dass „Männlichkeit“ wie auch „Weiblichkeit" als „diskursives Konstrukt" (Stephan, 2003, S. 13) gesehen werden und unmittelbar mit dem gesellschaftlich kulturellen Kontext interagieren: „Beide Konstrukte reagieren einerseits auf die massiven gesellschaftlichen Umbrüche um 1900, wie sie andererseits die ideologischen Muster bereitstellen, auf denen sich westliche Gesellschaften bis heute formieren" (Stephan, 2003, S. 13). Offensichtlich ist diese Auffassung über Männlichkeit rückführbar auf die Erkenntnisse der Geschlechterforschung, wie sie etwa von Judith Butler etabliert wurde. In ihrer bahnbrechenden Untersuchung Das Unbehagen der Geschlechter macht Butler anhand ihres kritischen Streifzugs durch die grundlegenden Theorien des Feminismus und der Psychoanalyse erkennbar, dass sowohl das Geschlecht als auch die Geschlechtsidentität keine ursprünglich gegebenen Kategorien sind, sondern „umgekehrt als Effekt jenes kulturellen Konstruktionsapparats verstanden werden" müssen (Butler, 2003, S. 24). Butlers Thesen waren somit auch der Anstoß, „Männlichkeit und Weiblichkeit gleichermaßen als Variablen diskursiver Praktiken [...] und als Ergebnis komplexer Inszenierungsstrategien" (Stephan, 2003, S. 17) zu begreifen. In der jüngeren literaturwissenschaftlichen Forschung zur Männlichkeit argumentiert auch Toni Tholen ganz in diesem Sinne:

8 Auch Schößler verweist auf den Umstand, dass Freud „störende Aspekte der Männlichkeitsentwicklung auf das Weibliche [verschiebe] und [...] so Irritationen aus der Genese des Mannes aus[blende]" (2008, S. 141).

9 Für eine Darstellung der historischen Entwicklungslinien der in den USA etablierten Männlichkeitsforschung siehe Stephan (2003, S. 18-19) sowie Schößler (2008, S. 133-144). 
In der Männlichkeitsforschung herrscht weitgehend Einigkeit darüber, Männlichkeit nicht als wesenhaft und unveränderlich zu denken, sondern als soziale Konstruktion. Sie kann mithin genauer bestimmt werden als ein diskursives Konstrukt bzw. als eine Konfiguration sozialer, historisch variabler kultureller Praktiken und Bilder. (Tholen, 2015, S. 11)

Sofern man vor diesen dargestellten Argumentationen zur diskursiven Verfasstheit von Männlichkeit die Männerfiguren aus den Romanen Imperium von Kracht und Risiko von Kopetzky betrachtet, so wird zunächst feststellbar, dass die Konfiguration von Männlichkeit im unmittelbaren Bezug zu den politischen sowie kulturellen Praktiken bzw. Ideologien des deutschen Kolonialismus steht. Im folgenden Abschnitt wird es darum gehen, die in diesen Romanen hervortretenden Männerfiguren als eine Form von hegemonialer Männlichkeit lesbar zu machen und dabei deren diskursive Verknüpfung zu der imperialen Weltpolitik Deutschlands zu veranschaulichen. Leitend für meine Argumentation ist hierbei eine genderorientierte und "männlichkeitssensible“ Lektüre, die im Anschluss an Tholen darauf bedacht ist, "die Vielfalt und damit auch Ambivalenz und Ambiguität von Geschlechterbildern" aufzuspüren, gleichzeitig eine "kritische Reflexion" einer "normativ statuierten Männlichkeit“ (Tholen, 2015, S. 12-13) zu leisten und auf diese Weise die heterogene Verfasstheit von männlichen Figuren innerhalb der Literatur freizulegen.

\section{Die Konfiguration „hegemonialer Männlichkeit“}

Die imperiale Weltpolitik von Wilhelm II. war vornehmlich auf die Ausweitung und Vergrößerung Deutschlands bedacht. Denn nach der Gründung des Deutschen Reichs 1871 versuchte sich Wilhelm II. innerhalb der imperialen Weltordnung dahingehend zu behaupten, dass er für eine intensivierte Kolonialpolitik eintrat. Die Befürworter der „Kolonialbewegung" „erhofften sich nicht nur ein Ventil für die angeblich drohende Überbevölkerung und einen Absatzmarkt für die wachsende industrielle Produktion, sondern auch ein sichtbares Symbol für die gewünschte Weltmachtrolle" (Zimmerer, 2013, S. 26) ${ }^{10}$. Der damalige Außenministier und spätere Reichskanzler Bernhard von

10 Entscheidend ist hierbei die Selbstwahrnehmung des "deutschen Bürgertum[s]", das sich „innerhalb der europäischen Nationen" wie auch „im Vergleich zu außereuropäischen Kulturen“ prononciert als "überlegener" sah (Zimmerer, 2013, S. 26-27). Die kolonialen Ambitionen fanden demnach insofern eine Legitimationsgrundlage, als die Deutschen die „Kultivierung der vermeintlich zurückgebliebenen und primitiven Bewohner der außereuropäischen Welt" (Zimmerer, 2013, S. 27) als eine Verpflichtung im Namen der Zivilisation sahen. 
Bülow führte denn auch in seiner historischen Rede die Wendung vom „Platz an der Sonne" ein und plädierte damit eindringlich für eine expansionistische Weltpolitik: „Mit einem Worte: wir wollen niemand in den Schatten stellen, aber wir verlangen auch unseren Platz an der Sonne" (Bülow, 2020, S. 2; vgl. auch Zimmerer, 2013, S. 27). Es war diese Expansionspolitik, welche die sogenannte "aggressive[] ,Weltpolitik' Wilhelms II." und eine damit verknüpfte "konservativ-nationalistische Weltsicht" einleitete, die „Imperialismus, Rassismus und Antisemitismus, Volkstümelei, Sozialdarwinismus, Kulturpessimismus und Militarismus amalgamierte" (Koller, 2017, S. 400). Diese proklamierte und ebenso die Politik bestimmende Selbstwahrnehmung der Deutschen ist weniger auf die historisch bedingte Erklärung zurückzuführen, dass es sich hier um eine „verspätete Nation“ handelte, vielmehr ging diese ambitionierte sowie offensive Politik, wie dies Honold und Simons festhalten, von einer "dezentrierten und exzentrisch agierenden“ (2002, S. 11) Haltung aus. Vor diesem historischen Hintergrund ist es bemerkenswert, dass Wilhelm II. als Staatsoberhaupt und Träger einer sich durch Aggression auszeichnenden Weltpolitik "zeitlebens unter einem besonderen Druck des Virilitätsnachweises stand“, was Inge Stephan zufolge auf die während seiner Geburt zustande gekommene körperliche Behinderung rückführbar sei und demzufolge die exorbitante bzw. auffällige mediale Selbstinszenierung als imperialer Herrscher begründe (2003, S. 15).

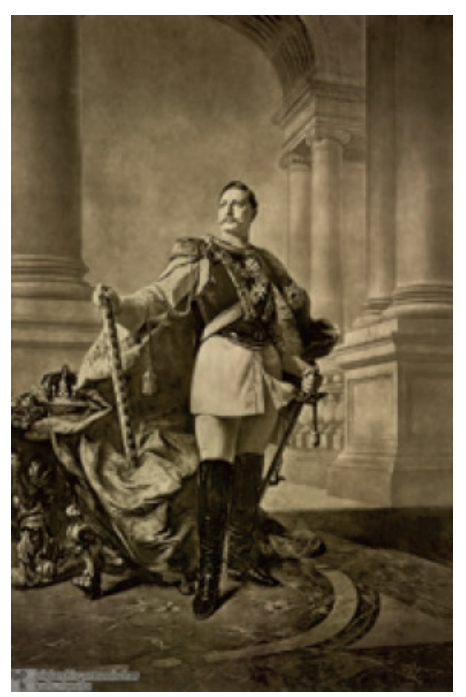

Abbildung 1. Kriegsverlust von Max Koner. (Deutsches Historisches Institut, 2020) 
Dieses angeführte Gemälde ${ }^{11}$ macht sichtbar, inwiefern Wilhelm II. um eine Idealisierung seines männlichen Ichs bemüht war. Dabei spielt das Militärische eine besondere Rolle, denn in „Preußen-Deutschland war der Militarismus [...] sehr viel markanter ausgeprägt als in anderen europäischen Staaten“, und der Kaiser "war durch seine Vorliebe für alles Militärische stilprägend“ (Epkenhans, Gross \& Köster, 2011, S. 174), zumal die Vorbildrolle und Selbstinszenierung des Kaisers als militärisches Vorbild besonders entscheidend war ${ }^{12}$.

Diese mit expansionistischer Ideologie und einer gezielten Inszenierung von militärischer Männlichkeit einhergehende Machtdimension im Bild Wilhelms II. spielt auch in den hier zu behandelnden Romanen auf je unterschiedliche Weise eine gravierende Rolle $^{13}$. In Imperium ist die Ausreise von August Engelhardt in die Südsee und die Erwerbung der Insel Kabakon in Neu-Guinea, um hier ein neues Reich der Kokosnuss zu errichten, geopolitisch betrachtet mit der Expansionsideologie Wilhelm II. kompatibel. Unterdessen zeigt sich in Risiko diese Ideologie in Form der Expedition einer deutschen Truppe nach Afghanistan, zwar nicht um dieses Land zu kolonisieren, wohl aber Afghanistan im imperialen Kampf gegenüber den Briten insofern zu instrumentalisieren, als durch ihre Hilfe die Muslime zu einem Dschihad aufgerufen werden sollten, um in letzter Konsequenz die in Indien vorherrschenden Briten zu besiegen. Insofern das kulturhistorische Assoziationsfeld von Wilhelm II. den diskursiven Rahmen beider Romane bildet, so kann argumentiert werden, dient dieses gleichzeitig als Vorlage einer "hegemonialen Männlichkeit" (Connell, 2012, S. 167). Dabei handelt es sich um eine innerhalb der soziologischen Männlichkeitsforschung von Connell eingeführte Beschreibungskategorie. Der Begriff ,hegemonial' macht offensichtlich, dass in dieser Einordnungspraxis die Komponente der Macht sowie die Zusammenführung von Macht und Männlichkeit eine unübersehbar wichtige Rolle spielt. Hierzu schreibt Connell Folgendes:

Das Konzept der "Hegemonie" stammt aus der Analyse der Klassenbeziehungen von Antonio Gramsci und bezieht sich auf die gesellschaftliche Dynamik, mit welcher eine Gruppe eine Führungsposition im gesellschaftlichen Leben einnimmt

11 Das Gemälde mit dem Titel „Kriegsverlust“ ist von Max Koner (vgl. Deutsches Historisches Institut, 2020).

12 Die Selbstinszenierungen von Wilhelm II. wurden damals auch im Osmanischen Reich insofern wahrgenommen, als der Wilhelm-Bart des Kaisers innerhalb der mit Deutschland sympathisierenden Kreise als Mode empfunden und imitiert wurde.

13 Ganz im Sinne von Tholen bietet es demnach an, „die literarische Männlichkeitsgeschichte" der männlichen Figuren beider Romane „mit der Geschichte der Männlichkeit im (historischen und kulturellen) Kontext einer Epoche zusammenzudenken“ (Tholen, 2015, S. 17). 
und aufrechterhält. Zu jeder Zeit wird eine Form von Männlichkeit im Gegensatz zu den anderen kulturell hervorgehoben. Hegemoniale Männlichkeit kann man als jene Konfiguration geschlechtsbezogener Praxis definieren, welche die momentan akzeptierte Antwort auf das Legitimitätsproblem des Patriarchats verkörpert und die Dominanz der Männer sowie die Unterordnung der Frauen gewährleistet (oder gewährleisten soll). (Connell, 2012, S. 167)

Bemerkenswert ist jedoch, dass nicht "direkte Gewalt" diese „Hegemonie" kennzeichnet, sondern vielmehr ein „erfolgreich erhobene[r] Anspruch auf Autorität” sowie die Vorbildrolle einer Person (Connell, 2012, S. 167). Zweifelsfrei übernimmt Wilhelm II. als Staatsoberhaupt mitsamt der akzentuierten militärisch formierten Männlichkeit eine explizite „Führungsposition“ und dient als dominierendes „zeit- bzw. epochenspezifische[s] Leitbild“ (Tholen, 2015, S. 38), was bereits in der Bezeichnung dieser Zeit als Wilhelminische Ära seinen Niederschlag findet. Diese Denkfigur der hegemonialen Männlichkeit kann gewissermaßen in jenen Narrativen ausfindig gemacht werden, in deren Mittelpunkt die Abenteuer bzw. Erfahrungen eines männlichen Helden stehen. Das bedeutet also, dass sich die Gattung des Abenteuerromans - sowohl Imperium als Risiko können mitunter dieser Gattung zugeordnet werden - für die Übertragbarkeit dieses soziologisch fundierten Konzepts auf die Analyse von literarischen Männlichkeitsentwürfen besonders anbietet. Horkheimer und Adorno zeigen in ihrer bahnbrechenden Schrift Dialektik der Aufklärung auf, inwiefern Homers Held Odysseus ${ }^{14}$ das „Urbild [...] des bürgerlichen Individuums" exponiert und vornehmlich durch "einheitliche[] Selbstbehauptung" (Horkheimer \& Adorno, 2006, S. 50) gekennzeichnet ist. Zugleich verweisen Horkheimer und Adorno auf ein markantes Merkmal der Heldenstruktur, das darin besteht, dass "Odysseus, wie die Helden aller eigentlichen Romane nach ihm, [...] sich weg[wirft] gleichsam, um sich zu gewinnen" (Horkheimer \& Adorno, 2006, S. 55). So gesehen, sind Narrative über Helden vornehmlich durch die Komponenten der "einheitlichen Selbstbehauptung" sowie des Selbsterhalts strukturiert und proklamieren demnach implizit die Verschränktheit von Macht und Männlichkeit. Offensichtlich kann somit Odysseus als prototypische Heldenfigur ebenso als Urbild von hegemonialer Männlichkeit bezeichnet werden. Zieht man in Betracht, dass Krachts und Kopetzkys Romane als Abenteuerromane angelegt sind, denen dem Modell des Abenteuerromans entsprechend Phänomene wie der "Ausbruch“ der männlichen Figuren "aus einer festgefügten Ordnung in eine unbekannte, als fremd erfahrene Welt", "Zeitkritik" und „das Interesse an der Ethnographie neu erschlossener Erdteile" (Grimm,

14 Homers Odyssee wird in der Tat als Vorform des Abenteuerromans gesehen. Vgl. dazu auch Dunker (2009, S. 3). 
2007, S. 2) immanent sind, so kann festgehalten werden, dass es sich vor allem um eine mit Männlichkeit korrespondierende Gattung handelt. Dies macht evident, in welchem Ausmaß "die literarische Form" für die literarische „Konstruktion“ von Männlichkeit einen maßgebenden Faktor bildet (Tholen, 2015, S. 18).

Untersucht man vor diesem Hintergrund die Hauptfigur August Engelhardt aus Imperium, so kann in dieser Figur auf den ersten Blick ein von diesem Konzept der hegemonialen Männlichkeit abweichender Männlichkeitsentwurf konstatiert werden ${ }^{15}$. Denn Engelhardt zeichnet sich in erster Linie durch eine ausdrückliche oppositionelle Haltung gegenüber den wirtschaftlichen, politischen und moralischen Interessen seiner Gesellschaft aus, so dass sein Entschluss, „dieser vergifteten, vulgären, grausamen, vergnügungssüchtigen, von innen heraus verfaulenden Gesellschaft, die lediglich damit beschäftigt ist, nutzlose Dinge anzuhäufen, Tiere zu schlachten und des Menschen Seele zu zerstören“ (Kracht, 2012, S. 92), endgültig feststeht. Er möchte „Pflanzer" werden, ,'doch nicht aus Profitgier, sondern aus zutiefst empfundenem Glauben, er könne Kraft seiner großen Ideen die Welt, die ihm feindlich, dumm und grausam dünkte, für immer verändern" (Kracht, 2012, S. 19). Obgleich also die kritische und negierende Haltung der historisch-fiktionalen ${ }^{16}$ Hauptfigur August Engelhardt gegenüber der politischgesellschaftlichen Atmosphäre dieser Zeit die Ausreise in die Südsee begründet, ist es doch beachtenswert, dass gerade in der Konzipierung dieser Figur diskursive Muster wiederzufinden sind, die aufs Engste mit dem hegemonialen Männlichkeitsideal von Wilhelm II. verknüpft sind:

August Engelhardts sehnlichster Wunsch, ja seine Bestimmung war es, eine Kolonie der Kokovoren zu erschaffen, als Prophet sah er sich und als Missionar zugleich. Aus diesem Grunde fuhr er in die Südsee, die schon unendlich viele Träumer gelockt hatte mit dem Sirenenruf des Paradieses. (Kracht, 2012, S. 20)

Anhand dieser zitierten Passage wird plausibel, dass die Südsee als geographischer Ort unmittelbar im Kontext der Kolonialpolitik zu sehen ist. Augenscheinlich intendiert er, eine Kolonie zu gründen; dabei ist die Selbstwahrnehmung als Prophet oder Missionar entscheidend, was offenbar auf seine hegemoniale Position hinweist. Denn alleine die

15 Ganz in diesem Sinne wird auch Dürbeck zufolge in Imperium „das Genre des historischen Reise- und Abenteuerromans" unterlaufen (2014, S. 110).

16 Zur metafiktionalen Darstellung dieses historischen Abenteuerromans siehe ausführlich Hauenstein (2014, S. 120-151). 
Entscheidung August Engelhardts ausgerechnet in die Südsee zu fahren, ist in diesem Zusammenhang vielsagend. Es handelt sich einerseits um eine mit dem Sirenenruf assoziierte Topographie - ein expliziter Verweis zu den Abenteuern von Odysseus - und andererseits tatsächlich um den hauptsächlichen Zielpunkt der kolonialen Expansionspolitik Deutschlands:

Die Deutschen Schutzgebiete im Stillen Ozean, hierin stimmten die Experten überein, waren, im Gegensatz zu den afrikanischen Besitzungen seiner Majestät Kaiser Wilhelm des Zweiten, allesamt vollkommen überflüssig. Der Ertrag der Kopra, des Guanos und des Perlmutts reichte bei weitem nicht aus, ein derart großes, in der Unendlichkeit des Stillen Ozeans versprenkeltes Reich zu unterhalten. Im fernen Berlin aber sprach man von den Inseln wie von kostbaren, leuchtenden Perlen, zu einer Kette aufgereiht. Fürsprecher und Gegner der pazifischen Kolonien fanden sich zuhauf, meist waren es jedoch die noch jungen Sozialdemokraten, welche die Frage nach der Relevanz der Südseebesitzungen am lautesten stellten. (Kracht, 2012, S. 18)

August Engelhardts „Kolonie der Kokovoren“ (Kracht, 2012, S. 20) dient zwar nicht unmittelbar dieser Expansionspolitik, zumal Engelhardt „kein politisch interessierter Mensch“ (Kracht, 2012, S. 78) ist. Sein „Sonnenorden“ (Kracht, 2012, S. 160-161) bzw. sein „nacktes kommunistisches Utopia“17, das die Kokosnuss als heiligste Frucht glorifiziert und als "das pflanzliche Abbild Gottes" (Kracht, 2012, S. 40) betrachtet, will die Menschen „Von jeglicher Zivilisationskrankheit" (Kracht, 2012, S. 161) heilen. Entscheidend ist in dieser zivilisationskritisch angelegten Idee des Sonnenordens vor allem das Motiv der Sonne, denn die Kokosnuss wird insbesondere aufgrund ihrer Nähe und Affinität zur Sonne verherrlicht ${ }^{18}$ : „Sie wuchs an höchster Stelle der Palme, der Sonne und dem lichten Herrgott zugewandt" (Kracht, 2012, S. 19), also „dem Himmel und der Sonne am nächsten“ (Kracht, 2012, S. 40). So gesehen, kann hier eine diskursive Analogie zu der Rede Bülows vom „Platz an der Sonne“ hergestellt werden (vgl. auch Schwarz, 2012, S. 4). Insofern wird auch die Idee vom Sonnenorden in gewisser Weise als eine Folie der Weltpolitik Wilhelms II. beschreibbar, der zwar dieser diametral gegenübersteht, aber insofern strukturelle Ähnlichkeiten dazu aufweist, als er ebenfalls von Selbst- und Größenwahn durchzogen ist. Hinzu kommt, dass August Engelhardts Habitus und Reaktionen zeitweilig das Gehabe des hegemonialen und überlegenen weißen Mannes implizieren:

17 Zu den utopischen Zügen des Romans vgl. die Untersuchung von Atasoy (2015).

18 Gleichzeitig impliziert dieses Motiv das nationalsozialistische Hakenkreuz und deutet demnach auf die tragischen Konsequenzen des imperialen Wahns hin. 
Eine eigene Insel zu besitzen, auf der in freier Natur die Kokosnuß wuchs und gedieh! Es war Engelhardt noch gar nicht vollständig ins Bewußtsein vorgedrungen, doch jetzt, da das kleine Boot vom offenen Ozean in das stillere, transparente Gewässer einer kleinen Bucht glitt, deren hellgezauberter Strand von majestätisch hochragenden Palmen umsäumt war, begann sein Herz auf und nieder zu flattern wie ein aufgeregter Sperling. Meine Güte, dachte er, dies war nun wirklich seins! Dies alles! (Kracht, 2012, S. 65)

Unverkennbar macht diese herangezogene Textstelle evident, in welchem Ausmaß die Figur August Engelhardt trotz ihrer vehementen Kritik gegenüber ihrer Zeit den Besitz über die Insel ,genießt', so dass hier gleichsam die Brisanz des kolonialen Begehrens auf der Ebene der Figurenkonzeption zum Ausdruck kommt. So vergleicht der Text die Ankunft Engelhardts auf der Insel mit einer "Darstellung der Landung des Konquistadoren Hernan Cortés am jungfräulichen Strande von San Juan de Ulúa“ (Kracht, 2012, S. 66). Anhand dieser Analogiebildung scheint der Text eine explizite Beziehung zu den männlich und hegemonial kodierten Entdeckungs- sowie Abenteuernarrativen herzustellen und somit auch die Figur in diesen kulturgeschichtlichen Kontext zu stellen. Beachtenswert ist in diesem Zusammenhang, dass Engelhardt trotz seiner wahnhaften Ideen als „Pragmatiker" (Kracht, 2012, S. 55) agieren kann, um sich während seiner Werbung für eine Kolonie auf das Geschäft zu konzentrieren und seine Überzeugungen zu kaschieren. Entgegen seiner Absicht, sich als "gestrenger Inselkönig" (Kracht, 2012, S. 70) zu profilieren, zeigt und verhält sich Engelhardt indes nach dem Erwerb der Insel Kabakon als „Herr" (Kracht, 2012, S. 71) der Insel, deren Bewohner "augenblicklich die Autorität akzeptieren, mit der er ihnen das ab jetzt für sie geltende Kabakonsche Regularium nahebrachte" (Kracht, 2012, S. 73). Demnach kann seine Insel als eine Institution beschrieben werden, die im Grunde wie ein Staat funktionieren soll und der infolgedessen eine männliche Codierung immanent ist, da der Staat offensichtlich „eine männliche Institution“ (Connell, 2012, S. 163) darstellt. Es ist vor allem Engelhardts Beziehung zu dem Jungen Makeli, in der er als prototypischer weißer Mann in Erscheinung tritt, wenn er ihm Bücher in deutscher Sprache vorliest und somit nicht nur zu seiner Kultivierung beiträgt, sondern zudem seine kulturelle Überlegenheit als "Herr" zum Tragen bringt. In dieser Konstellation wird demnach Engelhardt als eine hegemoniale Figur konzipiert, was gleichzeitig die „geschlechterspezifische Konzeption des ,weißen Mannes' im Imperialismus" (Kessel, 2011, S. 376) sichtbar macht. Auch die Konzipierung seiner Behausung verstärkt diese Konstellation, denn es war „eher ein richtiges Haus [...], das Engelhardt sich hier inzwischen gebaut hatte" und das vielmehr von einem 
"geschickten Zimmermann" errichtet wurde (Kracht, 2012, S. 120). Die hegemoniale Verfasstheit der Hauptfigur Engelhardt macht sich überdies in zwei weiteren Beziehungen zu männlichen Figuren kenntlich, etwa zu Heinrich Aueckens, einem Vegetarier sowie Gleichgesinnten und Max Lützow, einem virtuosen Musiker aus Berlin. Während seines Inselaufenthaltes erhält Engelhardt Besuche aus Europa, und es sind vor allem die Besuche dieser beiden männlichen Figuren, die im Roman in den Vordergrund gestellt werden. Die Gesellschaft von Aueckens empfindet Engelhardt anfangs als sehr erheiternd, zumal er sich mit ihm über politische oder literarische Belange austauschen kann, da dieser „über einen ähnlichen Horizont verfügte" (Kracht, 2012, S. 117). Offenbar handelt es sich in dieser Figurenkonstellation um eine Beziehung zwischen zwei gleichermaßen ,zivilisierten' Männern und diese steht daher der asymmetrischen und hegemonial bzw. kolonial strukturierten Beziehung zu Makeli konträr gegenüber. Auch die Beziehung zu Max Lützow steht unter ähnlichen Vorzeichen, wobei diese Figur aufgrund ihrer künstlerischen Seite von Engelhardt zunehmend als Konkurrenz wahrgenommen wird, so dass er sich herausgefordert fühlt, sich als Besitzer der Insel wie auch des Sonnenordens zu behaupten. Engelhardt fragt sich, „wann der Musiker denn nun endlich die Kontrolle über Kabakon übernehmen würde“, und er betont, dass er „allein“ bestimme, „wohin es gehe" (Kracht, 2012, S. 185). Diese Attitüde Engelhardts lässt sich zweifelsfrei mit dem von Connell beschriebenen Anspruch auf Autorität engführen. Besonders die überheblich klingende Betonung der Eigeninitiative und Leitposition auf der Insel ist in diesem Sinne mit dem dominierenden Männlichkeitsideal dieser Zeit korrelierbar.

Auch in Kopetzkys Risiko kann das programmatische Streben der deutschen Imperialpolitik sowie die damit einhergehende Männlichkeitsvorstellung auf der Ebene der Figuren festgestellt werden. Dabei muss in Betracht gezogen werden, dass der Roman die deutsch-osmanische Expedition nach Afghanistan am Anfang des Ersten Weltkriegs fiktional erzählt. Es geht um einen Erzählgegenstand, der innerhalb des Krieges verortet ist. Entsprechend wird Männlichkeit unmittelbar mit Kampf, Krieg und vor allem Macht enggeführt. So gelangt Oskar Niedermayer, eine der wichtigen Figuren des Romans, im Hinblick auf die Ausführungen von Halford Mackinder, einem „weltpolitische[n] Analytiker" (Kopetzky, 2015, S. 225), zu folgender Quintessenz:

Mackinder hatte ein rein machtpolitisches Modell entworfen, für ihn war eine Armee, wofür er den Ausdruck manpower geprägt hatte, letztlich eine Recheneinheit. Eine Macht kämpfte gegen eine andere, und es gewann die mit der größeren Manpower. (Kopetzky, 2015, S. 225, Hervorhebung im Original) 
Als ein Anführer von Armeen und Expeditionen wird denn nun auch die Figur Oskar Niedermayer eingeführt. Der Text beschreibt diese Figur zwar als „respektabel“ (Kopetzky, 2015, S. 210), da sie die militärische Fähigkeit habe, die geplante Expedition nach Afghanistan anzuführen (vgl. Kopetzky, 2015, S. 222), jedoch gleichzeitig verweist er auf den diese Figur umgebenden "ganz unziemliche[n] Abenteuerodem“ (Kopetzky, 2015, S. 210). Nachdem Niedermayer den Auftrag für die Expedition nach Afghanistan von Max von Oppenheim erhält, besucht er Enver Pascha, den Kriegsminister des Osmanischen Reiches, den der Text als „allmächtig“ bezeichnet (Kopetzky, 2015, S. 296). Bemerkenswert ist dabei die Darstellung von Enver Pascha aus der Perspektive Niedermayers:

\begin{abstract}
Enver trug - dem deutschen Kaiser nachempfunden - einen auf beiden Seiten in Haken nach oben gehenden Schnurrbart und zeigte jenes feine Lächeln, das er auch auf Fotografien stets zur Schau trug. Aber nie hätte Niedermayer ihn so zierlich vermutet, mit fast damenhaft schmalen Händen. Man mochte sich kaum vorstellen, dass diese zarte Person als der begabteste Feldherr und beste militärische Organisator der zweiundzwanzig Millionen Untertanen des Sultans galt [...]. (Kopetzky, 2015, S. 298)
\end{abstract}

An dieser Textstelle geht es um das äußere Erscheinungsbild von Enver Pascha. Auf der einen Seite ist dabei der Schnurrbart entscheidend, denn dieser scheint als Zeichen dafür zu dienen, dass das von Kaiser Wilhelm II. präfigurierte Männlichkeitsbild ebenfalls im Osmanischen Reich rezipiert wurde. Gleichzeitig aber signalisieren auf der anderen Seite Begriffe wie „zierlich“, ",damenhaft" oder "zart" eine überlegene Haltung Niedermayers, die auf eine Verzerrung dieses „allmächtigen“ Mannes bedacht zu sein scheint. In dieser Wahrnehmungsweise macht sich demnach eine dem Orientalismus (vgl. Said, 1981) verpflichtete Perspektive kenntlich, da sich in den genannten Attribuierungen ein Codierungsanspruch geltend macht, der auf die eigene Überlegenheit und die Unterlegenheit des Gegenübers bedacht ist. Es ist gerade dieser Anspruch auf grenzenlose Überlegenheit, der ebenso das Kriegsgeschehen begründet. Denn die deutsche Flotte befindet sich im Mittelmeer und dies löst Unbehagen auf britischer Seite aus, der hauptsächlichen Konkurrenz aus deutscher Sicht (vgl. Kopetzky, 2015, S. 130). Der Anspruch auf Überlegenheit und Macht, von der soeben die Rede war, macht sich überdies in der Romanhandlung bemerkbar, als sich die Lage der Kriegspolitik verschärft, nachdem die Breslau unter der Führung von Souchon als russische Flotte getarnt ein französisches Schiff am Hafen von Bône anfeuert (vgl. Kopetzky, 2015, S. 171-174). Die Deutschen, die „außer Österreich-Ungarn kein[en] Verbündete[n]“ haben und einen „totale[n] Krieg gegen 
England, ohne Rücksicht auf Verlust" (Kopetzky, 2015, S. 145) riskieren, finden Zuflucht in Istanbul. Die fiktionale Darstellung der Afghanistan-Expedition im Roman macht dementsprechend sichtbar, dass diese Expedition in gewisser Hinsicht "der lebendig gewordene Alptraum englischer Weltpolitik“ ist und folglich „im Kern ein[] Angriff auf das heartland" (Kopetzky, 2015, S. 225, Hervorhebung im Original) darstellt. Mithilfe dieses die Grenzen der Vernunft überschreitenden Plans sehen die Deutschen ihre Aufgabe darin begründet, "Geschichte zu schreiben“ (Kopetzky, 2015, S. 434). Als die Teilnehmer der Expedition die Bagdadbahn besteigen, transportieren sie neben den Flugblättern zum Dschihad-Aufruf Waffen, Munition, Lebensmittel, Zelte und vor allem „eine nagelneue transportable Funkanlage mit allem Drum und Dran“ und "einem Generator" (Kopetzky, 2015, S. 379) mit sich. Die fiktionale Hauptfigur Sebastian Stichnote, aus deren Perspektive weitgehend das Geschehen geschildert wird, ist der Funker, der diese Anlage bedienen wird (vgl. Uysal Ünalan, 2019, S. 217). Jedoch hat weder er noch ein anderer „Gedanken an die Mühen verschwendet [...], welcher es bedürfen würde, die vier Tonnen schwere Anlage zu transportieren, wenn sie an der Endstation ihres Zuges angekommen wären und ihr ganzes Gepäck auf Lasttiere und Ochsenwagen umladen müssten“ (Kopetzky, 2015, S. 379). Mit Reflexionen dieser Art trägt der Roman zur Sichtbarmachung der irrationalen Facetten sowie Fragwürdigkeit der Expedition bei. Überdies wird erkennbar, dass die Afghanistan-Expedition unmittelbar mit den weltpolitischen Machtambitionen der Deutschen während des Ersten Weltkriegs korrespondiert und auf "Weltgeltung" (Kopetzky, 2015, S. 513) aus ist. Demnach wäre dieser Plan unter Bezugnahme auf Honold und Simons ebenfalls als "dezentriert" und „exzentrisch“ (2002, S. 11) zu beschreiben, unterdessen lassen sich die Akteure der Expedition im Sinne einer essentialistischen Definition von Männlichkeit mit "Risikofreudigkeit" oder "Unverantwortlichkeit" (Connell, 2012, S. 158) verknüpfen. In diesem Zusammenhang verkörpert die Figur Oskar Niedermayer eine „eiserne Disziplin“, kraft derer sie trotz aller Strapazen und Hindernisse auf das Ziel, d.h. das Vordringen nach Afghanistan fixiert bleibt:

\footnotetext{
Niedermayer selbst schien überall gleichzeitig zu sein, packte den einen oder anderen Tschawadars unsanft am Kragen und erhob drohend seine Reitpeitsche. Gegenüber seinen Offizieren hatte er keinen Zweifel daran gelassen, dass ihr Unternehmen, das nun vor seiner schwierigsten Bewährungsprobe stand, nur durch eiserne Disziplin zu bewerkstelligen sein werde. (Kopetzky, 2015, S. 536)
}

Auch als die Expedition im buchstäblichen Sinne zu Tode erschöpft ist und nicht weiter vorankommt, gelingt es ihm, die Leute vorwärts zu bewegen: „Wie er die Kraft 
dazu aufbrachte, blieb inm selber ein Rätsel. Vielleicht lag es daran, dass er nicht an den Rückweg dachte, sondern nur an den nächsten Marsch, den nächsten Kilometer, den nächsten Schritt" (Kopetzky, 2015, S. 591) Diese Männer-Figuren, die auf Besitz fokussiert sind und sich durch ein explizites Ambitioniert-Sein auszeichnen, werden offensichtlich zunächst als Repräsentanten eines hegemonialen bzw. „maskulinen Stereotyps“ greifbar, „das vor allem Charaktereigenschaften wie Mut, Willensstärke und Ehrgefühl sowie eine spezifische geistig-körperliche Ausprägung umfasst, welche in Zuschreibungen wie Mäßigung, Selbstkontrolle und in einem gesunden und wohlgeformten Körper Gestalt annimmt" (Tholen, 2015, S. 11). Die Gründung des Kokosnuss-Reichs auf der einen und die Expedition in den Orient auf der anderen Seite werden in diesem Zusammenhang als eine „männliche Konzeption der Welt“ (Said, 1981, S. 233) fassbar, innerhalb derer sich der weiße Mann dem ,Anderen' gegenüber stets hegemonial überlegen dünkt. Es ist jedoch vorzüglich der auf der Textebene beobachtbare Wandel dieser Figuren, welcher ein kritisches Neudenken des konventionell begründeten Männlichkeitsstereotyps ermöglicht. Denn beide Texte legen anhand der Kritik der von diesen männlichen Figuren verkörperten imperialen Weltpolitik Wilhelms II. die Narrativität von Männlichkeit frei. So sind die männlichen Figuren dieser Abenteuerromane insofern als Anti-Helden konzipiert, als ihr Vorhaben bezüglich des Kokosnuss-Reichs zum einen und der AfghanistanExpedition zum anderen scheitert. Im Folgenden soll demonstriert werden, dass in beiden Romanen eine Signifikanz des Körpers zu beobachten ist, anhand dessen die ambivalente Verfasstheit von Männlichkeit und zusammenhängend damit die Kritik an der Kolonialpolitik Deutschlands prononciert zum Tragen kommt.

\section{Die Signifikanz der Körperlichkeit}

Das von Odysseus, einem der frühesten literarischen männlichen Heroen, präfigurierte Konzept einer soliden und homogen gedachten Männlichkeit wird in den Romanen Imperium und Risiko vehement in Frage gestellt. Ausschlaggebend für den Abenteuerroman des 20. Jahrhunderts ist es, dass dieser "entweder als Ausdruck eines utopisch-revolutionären Impetus [...] oder einer affirmativ-regressiven Projektion“ (Grimm, 2007, S. 2) verstanden wird. Diese Besonderheiten sind insofern auf Imperium und Risiko übertragbar, als in diesen Texten erstens kritische Töne im Hinblick auf die Weltpolitik Wilhelms II. erklingen, zweitens innerhalb der erzählten Welt eine Verlagerung bzw. Reise in außereuropäische Gebiete vorliegt, drittens das koloniale Begehren des Wilhelminischen Deutschland das „utopisch-revolutionäre“ Anliegen der Figuren August Engelhardt und Oskar Niedermayer begründet und viertens dieses auf Fortschritt und 
Expansion bedachte koloniale Begehren im Endeffekt sich mit regressiven Projektionen wie Kannibalismus oder etwa die Reduktion auf den Körper Hand in Hand geht ${ }^{19}$. Obgleich in der Figur von Wilhelm II. die Stilisierung von militärischer und soldatischer Männlichkeit beobachtbar wird, ist es doch bemerkenswert, dass die vom Kaiser zur Schau getragene „Männlichkeit", so die Beobachtung von Inge Stephan, „immer wieder zu Spekulationen Anlass gab“ und gleichzeitig als,rätselhaft' empfunden wurde (2003, S. 15). Dieser Aspekt schlägt sich unmittelbar in der ebenso spekulativen, dezentrierten wie auch exzentrischen Verfasstheit der männlichen Figuren August Engelhardt, Oskar Niedermayer oder etwa Sebastian Stichnote nieder. Denn bezeichnenderweise codiert der Roman Imperium seinen Protagonisten als einen "Spinner" (Kracht, 2012, S. 161), während der Roman Risiko den Truppenführer der Afghanistan-Expedition Oskar Niedermayer als ein „Mitglied einer Geheimgesellschaft“ (Kopetzky, 2015, S. 380) und den Funker Sebastian Stichnote als opiumsüchtigen Teilhaber dieser geheimen Expedition konfiguriert.

Obgleich der Roman Imperium von Christian Kracht die Hauptfigur August Engelhardt als Besitzer der Insel Kabakon darstellt, sind im Text mitunter Szenen feststellbar, die neben der "hegemonialen" Verfasstheit die "untergeordnete" sowie „marginalisierte" (vgl. Connell, 2012, S. 168-170) Stellung dieser Figur deutlich machen ${ }^{20}$. So wird Engelhardt nicht nur aufgrund seiner Überzeugung bezüglich der Kokosnuss als marginal wahrgenommen, sondern dafür ist ebenfalls sein äußerliches Erscheinungsbild sowie sein Habitus entscheidend. Frau Emma Forsayth, mit der er über die Insel Kabakon verhandelt, genügt beispielsweise „ein Blick auf den mageren jungen Mann [...], um ihn als schüchternen, dem Leben etwas abgewandten Zeitgenossen einzuordnen" (Kracht, 2012, S. 57). Neben dieser Schüchternheit und der betonten Magerkeit sind es sein langer Bart, der sichtlich in Kontrast zu dem von Wilhelm II. steht, seine "asketische" Positur sowie seine „unmögliche Frisur" (Kracht, 2012, S. 61), die ihm ein exzentrisches Aussehen

19 Gerade in dieser Hinsicht können beide Romane im Hinblick auf die „Dialektik der Aufklärung" gelesen werden, insofern als sie das Potential der Regression, das in jeglichem Fortschrittsoptimismus (vgl. Horkheimer \& Adorno, 2006, S. 9-49) manifest ist, offenlegen und somit auf das Totalitäre und das damit einhergehende Barbarische der Wilhelminischen Politik hinweisen. Vor allem Imperium schließt mit einem Bild aus dem Ende des Zweiten Weltkriegs und enthält in diesem Sinne Implikationen bezüglich des Übergangs vom Wilhelminismus in die NS-Zeit. Auf diese Weise reflektiert der Roman die Entwicklung des Faschismus und Antisemitismus in Verbindung zu den Veränderungsprozessen seiner Hauptfigur Engelhardt mit. Zu den Anspielungen auf den Holocaust, die im Roman wiederzufinden sind, vgl. Moll (2017, S. 155-159) und auch Hauenstein (2014, S. 129-136 sowie S. 145-151).

20 Mit diesen Beschreibungskategorien versucht Connell, die Beziehungen zwischen männlichen Figuren zu erfassen. Tholen verweist in diesem Rahmen auf die Übertragbarkeit dieser Kategorien auf die literaturwissenschaftliche Praxis (vgl. 2015, S. 39). 
verleihen, so dass auch die Erzählinstanz diese Figur mit einem "Spatz" (Kracht, 2012, S. 61) vergleicht. Bemerkenswert ist darüber hinaus, dass der Roman diese Figur vielfach mit Lächerlichkeit ${ }^{21}$ kombiniert. Bereits während seiner Schiffsreise nach Neuguinea wird er aufgrund seiner vegetarisch orientierten Essgewohnheiten ausgelacht (vgl. Kracht, 2012, S. 25). Aber auch bei einer Zugfahrt, davon berichtet der auktoriale Erzähler, lachen ihn mitfahrende Kinder wegen seines Aussehens aus (vgl. Kracht, 2012, S. 78). Ebenso wird er in jener Szene ausgelacht, als er zum ersten Mal mit den Inselbewohnern in Kontakt tritt und in der Blutlache nach der Schlachtung eines Tieres ausrutscht (vgl. Kracht, 2012, S. 71). Als Engelhardt nach Herbertshöhe, dem Zentrum von DeutschNeuguinea reist, wird er wie folgt dargestellt: „Die wenigen Franzosen, die ihn nicht vollends ignorierten, hielten ihn für einen dem Primitivismus frönenden Kunstmaler [...], ergo für eine durchweg lächerliche Figur [...]" (Kracht, 2012, S. 134). Diese explizite Gleichsetzung der Hauptfigur mit Primitivismus einerseits und Lächerlichkeit andererseits ist als Indikator zu bewerten, dass Engelhardt gewissermaßen als störend und marginal dargestellt wird und gerade daher dem Bild der dominierenden Männlichkeit dezidiert widerspricht. Demnach kann in dieser Konfiguration eine Form von hierarchischer "Unterordnung" gesehen werden, denn es handelt sich, um auf die Beschreibungskategorien von Connell zurückzugreifen, unverkennbar um eine „Marginalisierung" Engelhardts, die aufgrund der an dieser zitierten Textstelle beobachtbaren „Ermächtigung hegemonialer Männlichkeit der dominanten Gruppe“ (Connell, 2012, S. 170), also der Franzosen, entsteht. Diese Marginalisierung wird gleichsam in der oben skizzierten Begegnung mit Frau Forsayth virulent, obwohl es sich hier um eine weibliche Figur handelt, die jedoch den kolonialen Diskurs repräsentiert und gerade daher Engelhardt gegenüber als hegemonial auftritt.

Dabei ist es die Signifikanz des Körpers, anhand dessen die Marginalisierung dieser männlichen Figur vernehmbar wird. Die im Roman brisant werdende Körperlichkeit kommt zunächst in Verbindung mit der zivilisationskritischen Weltauffassung Engelhardts zur Geltung, wenn dieser etwa seine Kleidung symbolhaft abfallen lässt, zunächst nur einen „Wickelrock“ (Kracht, 2012, S. 73) trägt und allmählich auch auf diesen verzichtet. $\mathrm{Zu}$ berücksichtigen ist an diesem Punkt, dass "Mode" ein Mittel ist, mit dem man Männlichkeit „konstruieren“ (Kessel, 2011, S. 376) kann. Die Männer des Bürgertums zeichneten sich durch ihren „grauen Anzug" aus und unterschieden sich somit nicht nur

21 In ihrer Untersuchung zu Imperium konstatiert Dürbeck, dass im Rahmen der "metafiktionale[n] Anlage" des Romans die Hauptfigur "wahlweise in ein positives oder negatives Licht getaucht und in diesem

Zusammenhang „der Lächerlichkeit preisgegeben“ wird (2014, S. 116). 
von den Adligen, sondern zugleich von „weiblich charakterisierten Männergruppen“, was letztlich auch bedeutet, dass diese Anzüge den männlichen Körper "entwirklichen" (Kessel, 2011, S. 376-377) und auf diese Weise der Gleichsetzung von Männlichkeit und Körperlichkeit entgegenwirken. Indem jedoch Engelhardt infolge seiner nudistischen Überzeugungen seinen Körper zur Schau trägt und sichtbar macht, reagiert er gegen eine solche Entwirklichung des männlichen Körpers und dekonstruiert in gewisser Hinsicht die durch die männliche Bekleidung konstruierte Männlichkeit ${ }^{22}$. Ein weiterer Aspekt, der mit der im Roman beobachtbaren Körperlichkeit in Bezug gesetzt werden kann, ist die "Auto-Anthropophagie“ (Kracht, 2012, S. 151). In diversen Szenen des Romans wird kenntlich, dass die Hauptfigur eine bemerkenswerte Hinwendung zum eigenen Körper erfährt. So versucht er beispielsweise, „etwas Schorf von seinem Schienbein zu pulen und heimlich in den Mund zu schieben" (Kracht, 2012, S. 127), oder knabbert "seine Fingernägel“ an, was der auktoriale Erzähler bereits als eine "kleine Form der Auto-Anthropophagie" (Kracht, 2012, S. 151) kommentiert. Ebenso ist sein Verlangen bezeichnend, das Sekret aus dem Ohr in den Mund zu führen (vgl. Kracht, 2012, S. 172). Dies findet seinen Höhepunkt darin, als er den eigenen Daumen verzehrt (vgl. Kracht, 2012, S. 216). In diesem Zusammenhang ist ebenfalls das Daumenlutschen zu bewerten, welches der Text folgenderweise beschreibt: „[...] unversehens schob er den rechten Daumen in den Mund und lutschte daran. [...] In einem Hohlraum des Selbst versinkend, erlaubte ihm das Saugen am Daumen, die Umwelt beinahe lückenlos auszublenden [...]" (Kracht, 2012, S. 133). Ähnlich wie das Verlangen, die eigene Körperflüssigkeit in den Mund zu führen, wird das Daumenlutschen offensichtlich als ein regressiv-infantiles Verhaltensmuster greifbar, das der "bürgerliche Mann des Wilhelminismus" (Theweleit, 2009, S. 426) dezidiert bekämpfen würde, da dieser den „Körper als Schmutz" (Theweleit, 2009, S. 425) begreift und mit Weiblichkeit assoziiert. Diese im Text akzentuierte und mit Auto-Anthropophagie verknüpfte infantile und triebhafte Affinität zum Körper, so kann argumentiert werden, dient demnach zur Dekonstruktion der von Wilhelm II. präfigurierten sowie entkörperlichten Männlichkeit ${ }^{23}$.

Die Reduktion des Mannes auf Körperlichkeit wird ebenso in einer Szene erkennbar, als die Hauptfigur August Engelhardt den anfangs Gleichgesinnten Aueckens, der im Text als „erstklassiger Mistkerl“ (Kracht, 2012, S. 115) definiert wird, tötet, nachdem dieser

22 Ute Frevert betont die Relevanz "der militärischen Uniform“, die vor allem zur Markierung und Prägung der Männlichkeit diene (2003, S. 277).

23 Theweleit stellt in seiner viel beachteten Darstellung Männerphantasien dar, wie die „Männerkörper" durch Panzer und soldatische Ausrüstung im Wilhelminismus konstruiert wurden (2009, S. 428). 
Engelhardts Freund Makeli vergewaltigt: „Wir sehen Aueckens erst tot wieder, bäuchlings und nackt am Boden liegend, mit zerschmettertem Schädel, etwas Gehirnmasse ist ausgetreten." (Kracht, 2012, S. 129) Unverkennbar koppelt der Text an dieser Stelle die idealisierte Lebensform Engelhardts, die wie bereits dargestellt als eine Projektionsfläche der Weltpolitik von Wilhelm II. fassbar wird, mit Gewalt und Barbarei. Diese Regression im Verhalten Engelhardts zeichnet sich gleichsam auch in seinem Aussehen ab, insofern er als "Schreckgespenst" (Kracht, 2012, S. 146) wahrgenommen wird. Seine Marginalisierung findet dementsprechend ihren Höhepunkt darin, dass er in eine "geistige Archaik" (Kracht, 2012, S. 189) versinkt und die Kontrolle über sein Bewusstsein, seinen Körper sowie sein Verhalten verliert ${ }^{24}$ :

Es ist nicht mit Sicherheit zu sagen, ob seine Diät oder aber seine zunehmende Einsamkeit als Ursache für die sich langsam anbahnende Seelenstörung anzusehen war, zumindest aber potenzierte der ausschließliche Verzehr von Kokosnüssen eine bei ihm schon immer vorhandene Irritabilität, eine Unruhe angesichts bestimmter, vermeintlich unveränderbarer, ihn vexierender äußerer Umstände." (Kracht, 2012, S. 136)

Dieser Abschnitt aus dem Roman veranschaulicht den irritierten sowie unruhigen Zustand der Hauptfigur, was den Verlust seiner hegemonialen Position impliziert ${ }^{25}$. Engelhardt verwandelt sich gar zum Antisemiten und verschwindet, bis er schließlich nach dem Ende des Zweiten Weltkriegs von amerikanischen Soldaten in einem verwilderten und animalischen Zustand entdeckt wird. Bemerkenswert ist auch der Umstand, dass weitere männliche Figuren aus Krachts Roman wie Heinrich Aueckens oder Max Lützow eine Form von Männlichkeit exponieren, die mit marginaler Männlichkeit zusammengedacht werden kann. Die Figur Lützow wird folgendermaßen beschrieben:

Max Lützow, Geigen- und Klaviervirtuose aus Berlin, Leiter des nach ihm benannten Lützow-Orchesters und blondhäuptiger Frauenheld [...]. Lützow war ausgebrannt, will sagen, erledigt; er war zivilisationsmüde und führte eine erschreckende Ansammlung halbimaginierter Krankheiten mit sich, derer er sich freimütig bedient

24 Gerade in dieser Verwandlung der Hauptfigur zeichnet sich Hauenstein zufolge sinnbildlich „die große Katastrophe des 20. Jahrhunderts" (2014, S. 148) ab.

25 Angesichts dieses Zustands bemerken Stauffer und Weyand, dass Engelhardt „letztlich unfähig [ist], seine Kokosnuss-Plantage rentabel zu bewirtschaften und eine funktionierende Kolonie aufzubauen" und "am Ende [...] ihn sogar noch sein Freitag Makeli [verlässt]“ (2017, S. 56). 
hatte, um die Malaise seines deutschen Alltags mit dem Tuche der Hypochondrie zu verhüllen. Er litt abwechselnd, je nach Wetterlage und Tagesform, unter Asthma, Rheumatismus, Keuchhusten, Migräne, Ennui, Schüttelfrost, Anämie, Schwindsucht, Ohrensausen, Knochenschwund, Rückenschmerzen, Würmern, Lichtempfindlichkeit und Dauerschnupfen. (Kracht, 2012, S. 148-149)

Nimmt man diese vermeintlichen Krankheitsmuster, d.h. die Hypochondrie als charakterisierende Eigenschaft dieser Figur in den Blick, so kann festgehalten werden, dass hier genau das Gegenbild einer hegemonialen Männlichkeitskonzeption auf den Plan gerufen wird. Dabei scheinen insbesondere die Zivilisationsmüdigkeit und der damit einhergehende Ennui, d.h. die Lebensmüdigkeit bzw. der Überdruss insofern entscheidend zu sein, als diese eine kritische Perspektive auf das dominierende Männlichkeitsideal darstellen.

Eine diesem Verfahren vergleichbare Reflexionsstruktur kann auch in Kopetzkys Risiko konstatiert werden. Zunächst bedarf in diesem Zusammenhang die Hauptfigur Sebastian Stichnote einer näheren Betrachtung. Als der jüngste von drei Brüdern wird er in Bayern von einer Amme großgezogen, da seine Mutter bei seiner Geburt ums Leben kommt. Er ist der Erste in der Familie, der "eine weiterführende Schule" (Kopetzky, 2015, S. 36) besuchen darf und später als Funker der Kaiserlichen Marine beitritt (vgl. Kopetzky, 2015, S. 37). In München besucht er an der Universität einige Vorlesungen, die ihn begeistern: „Sebastian brannte für die Welt der Physik, die ihm eine Sprache schien, in welcher die Menschheit mit dem Planeten selbst und dem Universum im Ganzen zu kommunizieren vermochte." (Kopetzky, 2015, S. 37) Dieses Interesse für das Metaphysische verbindet ihn gewissermaßen mit dem Meer, das ihm folgende Gefühle vermittelt:

Weshalb er es auch so liebte, an Deck zu stehen, wenn sie mitten auf hoher See waren und man am glimmenden Horizont die Rundung der Erde erahnte, jenes DaSein der Welt, an welches zu denken sie einem fast schon brachte, so dass man durch diese Substanz gleichsam überall war. Sehnsucht hieß sie. Ein ozeanisches Gefühl. (Kopetzky, 2015, S. 38)

Dieses ozeanische Gefühl assoziiert psychoanalytisch betrachtet regressive Tendenzen, die mitunter mit dem Unterbewusstsein korrelieren ${ }^{26}$. Es ist in diesem Zusammenhang beachtenswert, dass Stichnote den Sinn der Afghanistan-Expedition

26 Zur psychoanalytischen Erläuterung des „ozeanischen Gefühls“ vgl. Freud (1999b, S. 424-431). 
hinterfragt und während dieser Expedition an den Rand des Wahnsinns gerät. Er beginnt nämlich aufgrund seiner nicht aushaltbaren Zahnschmerzen Opium zu konsumieren, als die vom Opium ausgehende Betäubung nicht nur seine Zahnschmerzen, sondern auch die Qualen seiner Seele lindert, konsumiert er immer mehr Opium und wird süchtig danach. Die Torturen, die er während dieser Expedition aushalten muss, finden in den folgenden Zeilen ihren Niederschlag:

Er war ein Funker in der Funkerhölle und musste seine Zeit damit verbringen, sie totzuschlagen. Gestrandet in einer vor Hitze glühenden Stadt, in der er sich so weit fort von allem fühlte, was ihm wichtig war, dass er manchmal verrückt zu werden glaubte. (Kopetzky, 2015, S. 520)

Einen Ausweg aus dieser „Funkerhölle" stellt für ihn offensichtlich der Konsum von Opium bereit. Dass der Roman Stichnote als opiumsüchtige Figur modelliert, scheint ein Indikator für die mit dieser Figur kompatiblen kritischen Reflexionsebene im Hinblick auf das Ausmaß und die Wirkungen des kolonialistischen Diskurses zu sein. Den Grad des Rauschzustandes dieser Figur macht indes die folgende Textstelle nachvollziehbar:

Der Alptraum eines gewöhnlichen Träumers wäre hier wohl zu Ende gewesen. Er hätte die Augen aufgeschlagen, verwirrt umhergeblickt und sich seiner Umgebung versichert. Doch das vom Schlafmohn in Gang gesetzte Träumen war von größerer Stabilität, der Träumer erlebte es, als führte er ein Eigenleben, das langsam, Rausch für Rausch, Traum für Traum und mit wachsender Dosis sein Haupt erhob, ein Dämon, der sich von den inneren Energien des Opiumrauchers nährte. (Kopetzky, 2015, S. 524)

Prägnant für die Selbsterfahrung Stichnotes ist fortan das Gefühl des Nichts (vgl. Kopetzky, 2015, S. 535), so dass er zwischen „Traum und Wirklichkeit" (Kopetzky, 2015, S. 537) gar nicht richtig zu trennen in der Lage ist. Die Situierung dieser Figur an der Grenze zwischen Traum und Wirklichkeit, d.h. in einem Schwellenraum, ist hier entscheidend, da diese Grenzsituation auf das Nicht-Artikulierbare sowie Unheimliche des kolonialen Begehrens hindeutet. Ähnlich wie Engelhardt verliert auch die Figur Niedermayer zeitweise die Kontrolle über die Expedition und auch gewissermaßen über ihr Bewusstsein:

Wollte er das ganze Unternehmen nicht vollends gefährden, mussten er und seine Leute sich aus der trägen Umklammerung des Nabobpalasts und seiner 
somnambulen Stimmung befreien und wieder in die Offensive gehen. (Kopetzky, 2015, S. 455, Hervorhebungen S.U.Ü.)

Es ist die in diesem Zitat zum Ausdruck kommende "somnambule Stimmung", die den Verlust der hegemonialen Überlegenheit, welche für diese Figur anfangs kennzeichnend war, konkretisiert. Gerade dieses Umschwenken indiziert dementsprechend die Destabilisierung des kolonialen Diskurses und macht dessen Verschränkung mit der Konfiguration der Männerfiguren evident. Dieser signifikante Verlust des Bewusstseins, der Disziplin sowie der Selbstkontrolle macht daher deutlich, dass während dieser Expedition, die zweifelsfrei als Akt bzw. Resultat der Wilhelminischen Weltpolitik zu betrachten ist, jene Maßstäbe gänzlich verschwinden, die das westliche Subjekt konstituieren und regulieren. Dieser Punkt präzisiert folglich die Ambivalenz des kolonialen Begehrens, das zum einen auf eine territorial fundierte Expansion aus ist, zum anderen jedoch eine Regression des westlichen Subjekts sowie dessen Reduktion auf Körperlichkeit begründet. Ähnlich wie in Imperium scheint die Abmagerung ein sichtliches Indiz dafür zu sein, dass die männlichen Figuren nicht durch ihre Überlegenheit bzw. Macht gekennzeichnet sind, sondern einzig durch ihren Körper. So erschrickt etwa die Figur Stichnote, "als er in der Dunkelheit erahnte, wie abgemagert er und seine Kameraden alle schon waren" (Kopetzky, 2015, S. 552). Aufgrund ihres Hungers sind diese Männer gar genötigt, einem Pferd den Gnadenschuss zu geben, um sich von dessen Fleisch ernähren zu können (vgl. Kopetzky, 2015, S. 551). Die Reflexionen über den männlichen Körper führen das männliche Subjekt demgemäß mit Krankheit und Tod zusammen:

Wenn es sie [die Expeditionsteilnehmer, S.U.Ü.] überkam, rissen sich die erkrankten Männer mit krampfartig herausgestreckter Zunge die Hosen herunter und entleerten sich stöhnend an Ort und Stelle. Manchmal fiel einer hinterrücks in das Gemisch aus schmierigem Kot und Blut und blieb eine Weile liegen, bis man ihm aufhalf. (Kopetzky, 2015, S. 542)

Anhand dieser zitierten Stelle aus dem Roman, in der die männlichen Figuren buchstäblich in ihren Exkrementen versinken, wird ersichtlich, dass die mit Macht und Überlegenheit assoziierten Männlichkeitsentwürfe der Wilhelminischen Zeit eine förmliche Reduktion auf das Körperliche bzw. Materielle erfahren, da die Beherrschung über den Körper vollkommen annulliert wird. Im Epilog des Romans heißt es in diesem Sinne: 
Gewiss waren viele Männer, als die Feindseligkeiten im August 1914 begannen, mit der festen Überzeugung ins Feld gezogen, da sei ein böser Erbfeind, den es zu schlagen gelte. Doch die Monate des Stellungskrieges hatten von dieser Auffassung bei den meisten nichts übrig gelassen - streckten doch alle im selben Verhängnis, von der Kriegsmaschinerie zum Menschenmaterial degradiert worden zu sein, und wohlwissend, dass es denen auf der anderen Seite ebenso erging. (Kopetzky, 2015, S. 717-718, Hervorhebungen S.U.Ü.)

Hier wird die Degradierung bzw. der Verfall einer machtbesessenen Männlichkeit, die im Begriff "Kriegsmaschinerie" ihren Ausdruck findet, beschrieben. Dabei wird Männlichkeit dahingehend konfiguriert, dass die materielle Verfasstheit des männlichen Körpers besonders akzentuiert wird. Indem der Roman also die hegemoniale Männlichkeit anhand des Körperdiskurses, entmännlicht' und marginalisiert, inszeniert er gleichsam Männlichkeit als ein „komplexes Gefüge" (Tholen, 2015, S. 39) und „vielgestaltiges Konstrukt" (Tholen, 2015, S. 13). In dieser Hinsicht wird im Roman anhand des Körperdiskurses das mit militärischer Ausrüstung konnotierte Männlichkeitskonzept dieser Zeit entkleidet und somit unterlaufen. Das Anliegen dieser männlichen Akteure der Expedition, Geschichte schreiben zu wollen, subvertiert der Roman mithin durch Vergleiche mit Tieren oder Leichnamen, zumal es den Teilnehmern der Expedition schlechter gehe als "Tieren“ oder diese auf ihren Sätteln "reitende[n] Leichname[n]" gleichen würden (Kopetzky, 2015, S. 603). So bildet die Beschreibung dieser Expedition als „winzig klein“ (Kopetzky, 2015, S. 604) einen völligen Kontrast zu ihrer anfänglichen Beschreibung als „dem großen Plan“ (Kopetzky, 2015, S. 224) im Büro von Oppenheim²7. Der Roman rekurriert ebenso auf den für koloniale Diskurse signifikanten TropenkollerSyndrom und variiert dieses im Hinblick auf die Konzipierung der männlichen Figuren. Dabei handelt es sich um „ein psychisches Phänomen“, das „auf den Größenwahn einiger Militärs [zurückgeführt wurde]“ und durch „exzessive ,Brutalitäten' gegenüber kolonialen Untertanen, Männern und Frauen, die mit der Peitsche mißhandelt worden waren" (Schwarz, 2002, S. 87), gekennzeichnet ist ${ }^{28}$. Diese "exzessive Brutalität" erfahren die Männerfiguren des Romans am eigenen Körper und gerade darin manifestiert sich auch die von beiden Romanen konfigurierteVerschiebung, um auf die Beschreibungskategorien

27 Siehe hierzu ausführlicher Uysal Ünalan (2019, S. 225-228).

28 Auch die Konzipierung der Figur Gilbert-Khan ist in diesem Zusammenhang erwähnenswert: Dieser englische Spion, der sich als indischer Prinz ausgibt und an der Expedition teilnimmt, bringt skrupellos mehrere Menschen um, da er davon ausgeht, dass diese seine Tarnung preisgeben werden. Insbesondere in diesen Mordszenen (vgl. Kopetzky, 2015, S. 408 und 613) wird ebenfalls das Ausmaß der Barbarei erkennbar, was unmittelbar auf die Mechanismen einer expansionistisch geprägten Kolonialpolitik bezogen ist. 
von Connell (2012, S. 168-170) zurückzugreifen, von einer anfangs hegemonial modellierten Männlichkeit zu einem untergeordneten bzw. marginalisierten Männlichkeitsentwurf. Denn während in „der kolonialen Situation [...] die Kolonialherren die Körper der Anderen zum Medium [machen], in das sich die koloniale Gewalt einschreibt" (Schwarz, 2002, S. 90), d.h. die Gewalt einer weißen und hegemonialen Männlichkeit, werden in Risiko, aber auch in Imperium $^{29}$ die Körper der mit kolonialem Begehren gekennzeichneten Männerfiguren zum kritischen Selbstreflexionsmedium jenes kulturellen bzw. politischen Machtapparates, der ausdrücklich von Wilhelm II. und seiner Weltpolitik repräsentiert wird.

\section{Schlussbemerkung}

Es ist bezeichnend, dass sowohl Imperium als auch Risiko vor dem historischen Hintergrund der kolonialen Interessen der Weltpolitik Deutschlands in der Zeit von Wilhelm II. die ambivalente Verfasstheit von Männlichkeit literarisch durchspielen. Denn ähnlich wie der „sonnenhungrige“ Wilhelm II., der sich „in einen düsteren Kriegsherren" (Stephan, 2003, S. 16, Hervorhebungen im Original) verwandelte, zeichnen sich die in dieser Untersuchung herausgearbeiteten Männerfiguren sowie ihre Lebenswelten dadurch aus, dass sie einem extremen Wandel unterliegen. Demnach konnte festgestellt werden, dass das von Wilhelm II. repräsentierte Macht- und Männlichkeitsideal als eine Form von männlicher Selbstbehauptung sowie hegemonialer Männlichkeit, dem das Militärische wie auch die Expansionspolitik zugrunde liegt, einen wichtigen Anknüpfungspunkt sowie Referenzrahmen des Erzählgegenstandes beider Romane darstellt, sowohl im Hinblick auf die Konfiguration der männlichen Figuren als auch auf ihre Handlungs- und Erfahrungsräume. August Engelhardts Utopie vom „Sonnenorden“ (Kracht, 2012, S. 161) schlägt in einen barbarischen Kannibalismus um und lässt sich in dieser Hinsicht als eine Antizipation des NS-Regimes lesen, indessen gelangen die an der Niedermayer-Expedition beteiligten Männer während ihrer Reise dahingehend an die Grenzen der menschlichen Existenz, dass sie eine erhebliche Reduktion auf das Körperliche erfahren. Die Männlichkeitsbilder, die durch beide Romane inszeniert und konfiguriert werden, stehen somit unmittelbar im Zeichen des ins Negative umschlagenden Kolonialismus-Diskurses und fungieren in gewisser Hinsicht als dessen Negativfolie. Gerade daher bieten diese Texte eine Blicköffnung auf die „komplexe[] Textur von Männlichkeitsnarrationen" in der Zeit des deutschen Kolonialismus und

29 In Bezug auf Imperium bemerkt auch Moll, dass „der Südseediskurs gegen den Kolonisator ausschlägt und zum Tropenhorror wird" (2017, S. 151). 
Imperialismus und versinnbildlichen auf diese Weise die "Ambivalenzen, Gewalt, Widersprüche, Rückfälle und Brüche" (Tholen, 2015, S. 8), die sich im kulturellen Machtdiskurs manifestieren. Somit lässt sich diese Arbeit als Beitrag zur literaturwissenschaftlichen Untersuchung von Männlichkeitsentwürfen verstehen, die gleichzeitig deren Bezüge zu den Erkenntniszielen von postkolonialen Fragestellungen deutlich macht ${ }^{30}$. Ebenfalls konnte gezeigt werden, dass sich in beiden Romanen die Kritik am Kolonialismus und an männlichen Machtparadigmen mit einem Körperdiskurs verschränkt, der wiederum entlang der männlichen Figuren zum Tragen gebracht wird. Festzuhalten ist in diesem Zusammenhang, dass beide Romane den kontextuellen Grundbedingungen des kolonialen Konstruktionsapparats entsprechend zunächst hegemoniale Männerfiguren konzipieren, allerdings die Hegemonie dieser Figuren insofern destabilisieren, als sie Männlichkeit mit Körperlichkeit engführen und insoweit diese als marginalisierte Männerfiguren lesbar machen. Anhand dieser Verlagerung der Subjektposition der männlichen Figuren wie auch der damit einhergehenden Dezentrierung bringen beide Romane eine Dynamik zur Geltung, der es gelingt, die negativen Facetten und Konsequenzen des deutschen Kolonialismus bzw. des „kolonialen Wahnsinns" (Schwarz, 2002, S. 89) auf fiktionaler Ebene zu visualisieren. Aus dieser Perspektive betrachtet unterlaufen die in den Romanen Imperium und Risiko erzählten Männergeschichten den Mythos des Kolonialismus, welcher auf Sieg, Eroberung und Besitz des Fremden bedacht ist, und erweisen sich in diesem Zusammenhang als kritische Narrationen, die das Stereotyp vom, weißen Mann' wie auch den damit unmittelbar verknüpften Diskurs des Kolonialismus fragwürdig machen.

\section{Begutachtung: Extern begutachtet.}

Interessenkonflikt: Es besteht kein Interessenkonflikt.

Finanzielle Förderung: Dieser Artikel ist im Rahmen meines Forschungsprojekts "Deutschsprachige Gegenwartsliteratur und Postkolonialismus. Männlichkeitsbilder und koloniales Begehren in Christian Krachts Imperium und Steffen Kopetzkys Risiko" entstanden, das an der Philosophischen Fakultät der Ege Universität zwischen 2016-2019 durchgeführt wurde (BAP-16-EDB-010).

Peer-review: Externally peer-reviewed.

Conflict of Interest: The author has no conflict of interest to declare.

Grant Support: This study is a part of my research project entitled "Deutschsprachige Gegenwartsliteratur und Postkolonialismus. Männlichkeitsbilder und koloniales Begehren in Christian Krachts Imperium und Steffen Kopetzkys Risiko", carried out at Ege University, Faculty of Letters between 2016-2019 (BAP: 16-EDB-010).

30 Zur Analyse von Männlichkeitsbildern im Zusammenhang von postkolonialen Ansätzen vgl. beispielsweise auch Rohners (2013) Lektüre des Romans Homo faber von Max Frisch. 


\section{Literaturverzeichnis}

Atasoy, İ. (2015). Ütopyanın Sonu. Hindistancevizi İmparatorluğu - Christian Kracht'ın Imperium Romanında

Ütopyacı Dürtüler. In Alman Dili ve Edebiyati Dergisi 34. Studien zur Deutschen Sprache und Literatur. 2015/2. Istanbul, 73-84.

Birgfeld, J. (2012). Südseephantasien. Christian Krachts "Imperium" und sein Beitrag zur Poetik des deutschsprachigen Romans der Gegenwart. In Wirkendes Wort 62, H. 3, 457-477.

Braun, Ch. v. \& Stephan, I. (2005). Einführung. Gender@Wissen. In Ch. v. Braun \& I. Stephan (Hrsg.). Gender@ Wissen. Ein Handbuch der Gender-Theorien (S. 7-45), Köln u.a.: Böhlau.

Bülow, B. von (2020). Rede über „Deutschlands Platz an der Sonne“. Band 5. Das Wilhelminische Kaiserreich und der Erste Weltkrieg, 1890-1918 Bernhard von Bülow über Deutschlands „Platz an der Sonne“(1897), 1-2. (Quelle: Stenographische Berichte über die Verhandlungen des Reichstags. IX. Legislaturperiode. V. Session. 1897/98. Bd. 1. Berlin 1898, 60.). Online unter: http://germanhistorydocs.ghi-dc.org/pdf/deu/607_Buelow_ Platz\%20an\%20der\%20Sonne_111.pdf (letzter Zugriff am 04.02.2020)

Butler, J. (2003). Das Unbehagen der Geschlechter. (Aus dem Amerikanischen v. Kathrina Menke). Frankfurt a.M.: Suhrkamp.

Connell, R.W. (2012). Der gemachte Mann. Konstruktion und Krise von Männlichkeiten. In F. Bergmann \& F. Schößler \& B. Schreck (Hrsg.). Gender Studies (S. 157-174). Bielefeld: transcript.

Deutsches Historisches Institut (2020). http://germanhistorydocs.ghi-dc.org/sub_image.cfm?image_id=2282 (letzter Zugriff am 04.02.2020)

Dietze, G. (2005). Postcolonial Theory. In Ch. v. Braun \& I. Stephan (Hrsg.). Gender@Wissen. Ein Handbuch der Gender-Theorien (S. 304-324). Köln u.a.: Böhlau.

Duden, Etymologie (1997). Herkunftswörterbuch der deutschen Sprache. Bearbeitet von G. Drosdowski. Nach den Regeln der neuen dt. Rechtschreibung überarbeiteter Nachdruck der 2. Auflage, Bd. 7. Mannheim u.a.: Dudenverlag.

Dunker, A. (2009). Abenteuerroman. In D. Lamping (Hrsg.). Handbuch der literarischen Gattungen (S. 1-8). Stuttgart: Alfred Kröner.

Dürbeck, G. (2014). Ozeanismus im postkolonialen Roman: Christian Krachts Imperium. Saeculum 64/I, 109-123. Epkenhans, M., Gross, G. P. \& Köster, B. (2011). Preussen. Aufstieg und Fall einer Grossmacht. Stuttgart: Theiss.

Freud, S. (1999a). Die Weiblichkeit (33. Vorlesung). In S. Freud. Gesammelte Werke in achtzehn Bänden mit einem Nachtragsband, hrsg. von Anna Freud u.a., Bd. 15: Neue Folge der Vorlesungen zur Einführung in die Psychoanalyse (S. 119-145), Frankfurt a.M.: Fischer Verlag.

Freud, S. (1999b). Das Unbehagen in der Kultur. In S. Freud. Gesammelte Werke in achtzehn Bänden mit einem Nachtragsband, hrsg. von Anna Freud u.a., Bd. 14: Werke aus den Jahren 1925-1931 (S. 419-506). Frankfurt a.M.: Fischer Verlag.

Frevert, U. (1995). „Mann und Weib, und Weib und Mann“. Geschlechter-Differenzen in der Moderne. München: C.H. Beck. 
Frevert, U. (2003). Männer in Uniform. Habitus und Signalzeichen im 19. und 20. Jahrhundert. In C. Benthien \& I. Stephan (Hrsg.). Männlichkeit als Maskerade. Kulturelle Inszenierungen vom Mittelalter bis zur Gegenwart (S. 277-295). Köln u.a.: Böhlau.

Grimm, G. E. (2007). Abenteuerroman. In D. Burdorf \& Ch Fassbinder \& B. Moennighoff (Hrsg.). Metzler Lexikon Literatur. Begriffe und Definitionen. 3., völlig neu bearbeitete Auflage (S. 1-2). Stuttgart/ Weimar: Metzler.

Hauenstein, R. (2014). Historiographische Metafiktionen. Ransmayr, Sebald, Kracht, Beyer. Würzburg: Königshausen \& Neumann.

Honold, A. \& Simons, O. (2002). Einleitung: Kolonialismus als Kultur? In Dies. (Hrsg.). Kolonialismus als Kultur. Literatur, Medien, Wissenschaft in der deutschen Gründerzeit des Fremden (S. 7-15). Tübingen/Basel: A. Francke.

Horkheimer, M. \& Adorno, T.W. (2006). Dialektik der Aufklärung. Philosophische Fragmente. Frankfurt a.M.: Fischer. Kessel, M. (2011). Heterogene Männlichkeit. Skizzen zur gegenwärtigen Geschlechterforschung. In F. Jaeger \& J. Rüsen (Hrsg.). Handbuch der Kulturwissenschaften. Bd. 3. Themen und Tendenzen (S. 372-384). Stuttgart/ Weimar: Metzler.

Kiepenheuer \& Witsch (2020). Verlagswerbung zu Christian Kracht Imperium. Online unter: https://www.kiwiverlag.de/buch/christian-kracht-imperium-9783462306019 (letzter Zugriff am 04.02.2020)

Klett Cotta (2020). Verlagswerbung zu Steffen Kopetzky Risiko. Online unter: https://www.klett-cotta.de/buch/ Gegenwartsliteratur/Risiko/55734 (letzter Zugriff am 04.02.2020)

Koller, C. (2017). Deutschland. Frühe Kolonialgeschichte (16.-18. Jahrhundert): Handel, Mission, Stützpunkte. In D. Göttsche \& A. Dunker \& G. Dürbeck (Hrsg.). Handbuch Postkolonialismus und Literatur (S. 399-402). Stuttgart: Metzler.

Kopetzky, S. (2015). Risiko. Stuttgart: Klett Cotta.

Kracht, C. (2012). Imperium. Köln: Kiepenheuer \& Witsch.

Moll, B. (2017). „Europavergiftung”. Südsee, Tropen und die Krankheit der Hochkultur in Christian Krachts Imperium. In P. M. Lützeler \& T. W. Kniesche (Hrsg.). Gegenwartsliteratur. Ein germanistisches Jahrbuch. A German Studies Yearbook 16/2017. Schwerpunkt/Focus:Daniel Kehlmann (S. 145-164). Tübingen: Stauffenburg. Rohner, M. (2013). „Wie ein Indio!“ Whiteness und non-whiteness in Max Frischs Homo faber. In A. Babka \& A. Dunker (Hrsg.). Postkoloniale Lektüren. Perspektivierungen deutschsprachiger Literatur (S. 111-130). Bielefeld: Aisthesis.

Said, E. W. (1981). Orientalismus. (Übersetzt von Liliane Weissberg) Frankfurt a.M.: Ullstein.

Schößler, F. (2008). Einführung in die Gender Studies. Berlin: Akademie Verlag.

Schumacher, E. (2015). Differenz und Wiederholung. Christian Krachts Imperium. In H. Winkels (Hrsg.). Christian Kracht trifft Wilhelm Raabe-Literaturpreis 2012 (S. 129-146). Berlin: Suhrkamp.

Schwarz, T. (2002). Die Kultivierung des kolonialen Begehrens - Ein deutscher Sonderweg?. In A. Honold \& O. Simons (Hrsg.). Kolonialismus als Kultur. Literatur, Medien, Wissenschaft in der deutschen Gründerzeit des Fremden (S. 85-103). Tübingen/ Basel: A. Francke. 
Schwarz, T. (2012). Eine Tragikomödie der Südsee. Marc Buhls und Christian Krachts historische Romane über das imperiale Projekt des August Engelhardt. In www.germanistik.ch. 1-15. Online unter: http://www. germanistik.ch/publikation.php?id=Eine_Tragikomoedie_der_Suedsee (letzter Zugriff am 07.04.2020)

Stauffer, I. \& Weyand, B. (2017). Antihelden, Nomaden, Cameos und verkörperte Simulakren. Zum Figureninventar von Christian Krachts Romanen. Text + Kritik. Zeitschrift für Literatur. H. 216. Christian Kracht, 54-66.

Stephan, I. (2003). Im toten Winkel. Die Neuentdeckung des „ersten Geschlechts" durch men's studies und Männlichkeitsforschung. In C. Benthien \& I. Stephan (Hrsg.). Männlichkeit als Maskerade. Kulturelle Inszenierungen vom Mittelalter bis zur Gegenwart (S. 11-35). Köln u.a.: Böhlau.

Theweleit, K. (2009). Männerphantasien. Bd. 1: Frauen, Körper, Geschichte. München, Zürich: Piper.

Tholen, T. (2007). Männlichkeit. In D. Burdorf \& Ch. Fasbender \& B. Moenninghoff (Hrsg.). Metzler Lexikon Literatur. Begriffe und Definitionen. 3., völlig neu bearbeitete Auflage (S. 472). Stuttgart/ Weimar: Metzler.

Tholen, T. (2015). Männlichkeiten in der Literatur. Konzepte und Praktiken zwischen Wandel und Beharrung. Bielefeld: transcript.

Uysal Ünalan, S. (2019). ,Subalterne Geschichtsschreibung' in dem Roman Risiko von Steffen Kopetzky: Eine postkoloniale Lektüre. Diyalog. Interkulturelle Zeitschrift für Germanistik. 7/2, 211-232. Online unter: https:// dergipark.org.tr/en/download/article-file/911040 (letzter Zugriff am 04.02.2020)

Uysal Ünalan, S. (Artikel in Vorbereitung). Kulturkritik und Reflexionen zum deutschen Kolonialismus in Christian Krachts Roman Imperium.

Vahsen, M. (2002). Männlich/ Männlichkeit/ Männlichkeitsforschung. In R. Kroll (Hrsg.). Metzler Lexikon. Gender Studies. Geschlechterforschung. Ansätze - Personen - Grundbegriffe (S. 252-253). Stuttgart, Weimar: Metzler.

Wendt, R. (2013). Die Südsee. In J. Zimmerer (Hrsg.). Kein Platz an der Sonne. Erinnerungsorte der deutschen Kolonialgeschichte (S. 41-55). Hamburg: Campus.

Winkels, H. (Hrsg.) (2015). Christian Kracht trifft Wilhelm Raabe-Literaturpreis 2012, Berlin: Suhrkamp.

Zimmerer, J. (2013). Kolonialismus und kollektive Identität: Erinnerungsorte der deutschen Kolonialgeschichte. In Ders. (Hrsg.). Kein Platz an der Sonne. Erinnerungsorte der deutschen Kolonialgeschichte (S. 9-38). Hamburg: Campus. 
